Pacific Journal of Mathematic 


\title{
TORSION DIVISORS ON ALGEBRAIC CURVES
}

\author{
GeORge R. KempF
}

\begin{abstract}
Let $D$ be a divisor on a smooth complete algebraic curve $C$ such that the multiple $d D$ is rationally equivalent to zero for some positive integer $d$. We may write $D=D_{0}-D_{\infty}$ where $D_{0}$ and $D_{\infty}$ are distinct effective divisors.
\end{abstract}

The purpose of this note is to prove the

THEOREM. Assume that $C$ is a curve with general moduli in characteristic zero. For such a divisor $D$, the cohomology $H^{1}(C$, $\left.O_{C}\left(D_{0}+D_{\infty}\right)\right)$ must be zero (equivalently $\left|K-D_{0}-D_{\infty}\right|$ is empty).

This theorem answers a question which arose in Sevin Recillas' unpublished work which motivated this note. This paper contains simple computational techniques for handling infinitisimal deformations of curves. I hope that these techniques may be useful for solving similar problems.

The theorem is well-known for the case $d=1$. For instance see Farkas' paper [2]. The recent work [1] contains further biliographic material and related material concerning deformations of mappings of curves into projective spaces.

1. First order deformations defined by principal parts. Let $\mathscr{L}$ be an invertible sheaf on a smooth curve $C$. For any point $c$ of $C, \operatorname{Prin}_{c}(\mathscr{L}) \equiv \operatorname{Rat}(\mathscr{L}) / \mathscr{L}_{c}$ measures the principal (polar) part of the rational sections of $\mathscr{L}$. Let $\operatorname{Prin}(\mathscr{L})$ be the space of principal parts, $\oplus \operatorname{Prin}_{c}(\mathscr{L})$, where $c$ runs through $C$. For a given principal part $p=\left(p_{c}\right)$ in Prin $(\mathscr{L})$, the support, $\operatorname{supp}(p)$, is the set of $c$ in $C$ with $p_{c} \neq 0$.

As in [3], we have a natural exact sequence

$0 \longrightarrow \Gamma(C, \mathscr{L}) \longrightarrow \operatorname{Rat}(\mathscr{L}) \longrightarrow \operatorname{Prin}(\mathscr{L}) \longrightarrow H^{1}(\mathrm{C}, \mathscr{L}) \longrightarrow 0$.

Let $p_{c}$ be a principal part in $\operatorname{Prin}_{c}(\mathscr{L})$ with a pole of order one. By duality the image of $p_{c}$ in $H^{1}(C, \mathscr{L})$ is zero if and only if each section of $\Omega_{C} \otimes \mathscr{L}^{\otimes-1}$ vanishes at $c$. Consequently, if $H^{1}(C, \mathscr{L}) \neq 0$ (i.e., there is a nonzero such section), the cohomology class of $p_{c}$ is nonzero for all but a finite number of points $c$.

Let $\Theta_{C}=\Omega_{C}^{\otimes-1}$ be the sheaf of regular vector fields on $C$. For any principal part $X$ in $\operatorname{Prin}\left(\Theta_{C}\right)$, we want to define a deformation $C_{X}$ of $C$ over $T=\operatorname{Spec}(k[\delta])$, where $k[\delta]$ is the ring of dual numbers $k[t] /\left(t^{2}\right)$. First note that, if $f$ is a rational function which is regular at each point of $\operatorname{supp}(X)$, then $X(f)$ makes sense in an obvious way 
as a principal part in $\operatorname{Prin}\left(O_{C}^{P}\right)$. Secondly define the structure sheaf of $C_{X}$ to be the subsheaf $\mathscr{A}_{X}$ of $\mathscr{O}_{C} \oplus \delta \mathscr{Y}$ (at $\left(\mathcal{O}_{C}\right)$, whose sections $f_{0}+f_{1} \delta$ locally satisfy the conditions: $f_{0}$ is regular and $X\left(f_{0}\right)$ is the principal part of $f_{1}$ (symbolically $(1-X \delta)\left(f_{0}+f_{1} \delta\right)$ is regular).

Locally on $C, X$ is the principal part of a rational vector field $\underline{X}$. For regular functions $g_{0}$ and $g_{1}$, the expression

$$
\phi_{\underline{X}}\left(g_{0}+\delta g_{1}\right) \equiv(1+\underline{X} \delta)\left(g_{0}+g_{1} \delta\right)=g_{0}+\left(\underline{X} g_{0}+g_{1}\right) \delta
$$

defines an $k[\delta]$-algebra isomorphism between $\mathscr{L}_{X}$ and $\mathcal{O}_{C} \oplus \delta \mathcal{O}_{C}$, which preserves the augmentations of the two algebras onto $O_{C}$. Therefore $C_{X} \rightarrow T$ is a (locally trivial) deformation of $C$ over $T$, which has been given an obvious trivialization over the open subset $C$-supp $(X)$.

ExAmPLE. If $t$ is a parameter function at a point $c$ and $t(c)=$ 0 , let $X$ be the principal part of the vector field $(1 / t)(d / d t)$ at $c$. This principal part is determined by $c$ upto constant. In this case, $C_{X}$ is called a (first-order) Schiffer variation of the curve $C$. (If one replaces $(1+X \delta)$ by $\exp (X t) \bmod t^{n}$, as $n \rightarrow \infty$ one gets the formal version of a Schiffer variation in the compact Riemann surface case.)

REMARK. The deformation $C_{X} \rightarrow T$ depends only on the cohomology class of $X$ in $H^{1}\left(C, \Theta_{C}\right)$. This class is a form of the KodairaSpencer class of the deformation.

2. Obstructions to a particular deformation problem. Let $D_{0}$ and $D_{\infty}$ be two effective divisors on our smooth curve $C$. Assume that $D_{0}$ and $D_{\infty}$ have disjoint support. We are interested in the following special situation. For some positive integer $d, d D_{0}$ is linearly equivalent to $d D_{\infty}$. In other words, there is a nonconstant morphism $f: C \rightarrow \boldsymbol{P}^{1}$ such that $f^{-1}(0)=d D_{0}$ and $f^{-1}(\infty)=d D_{\infty}$. We want to examine whether this special situation may be extended to a deformation of $C$.

Let $g: \widetilde{C} \rightarrow M$ be a deformation of $\widetilde{C}$. Thus $g$ is a smooth morphism and $C$ is a closed subscheme of $\widetilde{C}$, which has the form $g^{-1}(m)$ for some point $m$ of $M$. Then we may ask if we can find a morphism $\widetilde{f}: \widetilde{C} \rightarrow P^{1}$ such that $\left.\widetilde{f}\right|_{c}=f, \widetilde{f}^{-1}(0)$ and $\widetilde{f}^{-1}(\infty)$ are defined and we have $\widetilde{f}^{-1}(0)=d \widetilde{D}_{0}$ and $\widetilde{f}^{-1}(\infty)=d \widetilde{D}_{\infty}$ for some effective relative Cartier divisors $\widetilde{D}_{0}$ and $\widetilde{D}_{\infty}$ on $\widetilde{C}$ with $\left.\widetilde{D}_{i}\right|_{c}=D_{i}$ for $i=0$ or $\infty$.

Next we will analyze this concept for first order deformations. Let $C_{X} \rightarrow T$ be the deformation defined in the first section for a given principal part $X$ in $\operatorname{Prin}\left(\Theta_{C}\right)$. Here $C$ and $C_{X}$ are the same as topological spaces. Let $C_{i}$ denote the open subscheme $C$-supp $\left(D_{j}\right)$ of 
$C$ where $\{0, \infty\}=\{i, j\}$. Our original morphism $f$ is given by a pair $\left(f_{0}, f_{\infty}\right)$ where each $f_{i}$ is a regular function on $C_{i}$ and $f_{0} \cdot f_{\infty}=1$ on $C_{0} \cap C_{\infty}$. Similarly, our extension $\tilde{f}$ would be given by a pair $\left(f_{0}+f_{0}^{\prime} \delta, f_{\infty}+f_{\infty}^{\prime} \delta\right)$, where $f_{i}+f_{i}^{\prime} \delta$ is a section of $\mathscr{A}_{X}$ over $C_{i}$, such that

(*) $f_{0} f_{0}^{\prime}+f_{\infty}^{\prime} f_{\infty}=0$ on $C_{0} \cap C_{\infty}$. As the $f_{i}+f_{i}^{\prime} \delta$ are then reciprocals on $C_{0} \cap C_{\infty}$, the conditions on the zeros and poles of $\tilde{f}$ are just that, near $\operatorname{supp}\left(D_{i}\right)$,

(**) there exist sections $\alpha$ and $\beta$ of $\mathscr{A}_{X}$ with $f_{i}+f_{i}^{\prime} \delta=\alpha \beta^{d}$ and $\alpha$ is a unit.

In the simplest case, the question of the existence of such an $\tilde{f}$ is easily interpreted.

Proposition. Assume that $\operatorname{supp}(X)$ is contained $C_{0} \cap C_{\infty}$ and $\operatorname{char}(k) \nmid d$. Then the required extension $\tilde{f}$ exists if and only if the principal part $(1 / f) X(f)$ interpreted in $\operatorname{Prin}\left(O_{C}\left(D_{0}+D_{\infty}\right)\right)$ is cohomologous to zero.

Proof. By the assumption on the $\operatorname{supp}(X)$, any section of $\mathscr{A}_{X}$ near $\operatorname{supp}\left(D_{i}\right)$ can be written as $\alpha=\alpha_{0}+\alpha_{1} \delta$, where $\alpha_{0}$ and $\alpha_{1}$ are regular functions. Furthermore, this section $\alpha_{0}+\alpha_{1} \delta$ is a unit near $\operatorname{supp}\left(D_{i}\right)$ if and only if $\alpha_{0}$ is.

Claim. Given a rational function $f_{i}^{\prime}$ on $C$, then $f_{i}+f_{i}^{\prime} \delta$ is a section of $\mathscr{A}_{X}$ over $C_{i}$-supp $(X)$ and satisfies the condition $\left({ }^{* *}\right) \Leftrightarrow$ $\operatorname{div}\left(f_{i}^{\prime} / f_{i}\right)+D_{i} \geqq 0$ on $C_{i}$-supp $(X)$.

We will first see how this claim implies the proposition. Assume that $\left(f_{1}+f_{1}^{\prime} \delta, f_{\infty}+f_{\infty}^{\prime} \delta\right)$ gives a required $\widetilde{f}$. By the identity $\left({ }^{*}\right)$, we must have $f_{0}^{\prime} / f_{0}=-f_{\infty}^{\prime} / f_{\infty}$. Thus the claim shows that the desired conditions on the zeros and poles of $\tilde{f}$ imply that $f_{i}^{\prime} / f_{i}$ is a regular section of $\mathscr{O}_{C}\left(D_{0}+D_{\infty}\right)$ over $C$-supp $(X)$. As $f_{i}+f_{i}^{\prime} \delta$ is a section of $\mathscr{A}_{X}$ over $C_{i}, X\left(f_{i}\right)$ is the principal part of the rational function $f_{i}^{\prime}$ except at $\operatorname{supp}\left(D_{0}+D_{\infty}\right)$. So $f_{0}^{\prime} / f_{0}$ is a rational section of $\mathcal{O}_{c}\left(D_{0}+D_{\infty}\right)$ with principal part $1 / f_{0} X\left(f_{0}\right)$. Therefore, if our $\tilde{f}$ exists, $(1 / f) X(f)$ is cohomologous to zero in $\operatorname{Prin}\left(\mathscr{O}_{c}\left(D_{0}+D_{\infty}\right)\right)$.

Conversely, if $1 / f X(f)$ is cohomologous to zero, we may have a rational section $f_{0}^{\prime} / f_{0}$ of $\mathscr{O}_{c}\left(D_{0}+D_{\infty}\right)$ with principal part $1 / f_{0} X\left(f_{0}\right)$. Define $f_{\infty}^{\prime}$ by the identity $\left({ }^{*}\right)$. Reversing the above argument, one can readily check that the required extension $\tilde{f}$ is given by $\left(f_{0}+f_{0}^{\prime} \delta, f_{\infty}+f_{\infty}^{\prime} \delta\right)$. Thus the claim implies the proposition.

To prove the claim, assume that $f_{i}+f_{i}^{\prime} \delta$ satisfies condition (**). Then near $\operatorname{supp}\left(D_{i}\right)$ we have $f_{i}+f_{i}^{\prime} \delta=\left(\alpha_{0}+\alpha_{1} \delta\right)\left(\beta_{0}+\beta_{1} \delta\right)^{d}=\alpha_{0} \beta_{0}^{d}+$ 
$\left(\alpha_{1} \beta_{0}^{d}+d \beta_{0}^{d-1} \beta_{1} \alpha_{0}\right) \delta$, where the $\alpha$ 's and $\beta^{\prime}$ 's are regular and $\alpha_{0}$ is a unit. So $\beta_{0} f_{i}^{\prime} / f_{i}=\left(\alpha_{1} \beta_{0}+d \beta_{1} \alpha_{0}\right) / \alpha_{0}$ is regular. As $D_{i}=\left(\beta_{0}=0\right)$, this means that $\operatorname{div}\left(f_{i}^{\prime} / f_{i}\right)+D_{i} \geqq 0$ along $D_{i}$. Conversely, assume that this is ture. By the assumption on $f$, we have $f_{i}=\alpha_{0} \beta_{0}^{d}$, where $\alpha_{0}$ is unit and $D_{i}=\left(\beta_{0}=0\right)$ near $D_{i}$. Take $\alpha_{1}=0$ and $\beta_{1}=1 / d\left(\beta_{0} f_{i}^{\prime} / f_{i}\right)$. Thus $\beta_{1}$ is regular and condition (**) is satisfied for $\alpha=\alpha_{0}+\alpha_{1} \delta$ and $\beta=\beta_{0}+\beta_{1} \delta$ near $D_{i}$.

Away from $D_{i}$ on $\mathrm{C}_{i}-\operatorname{supp}(X), \operatorname{div}\left(f_{i}^{\prime} / f_{i}\right)+D_{i} \geqq 0 \Leftrightarrow f_{i}^{\prime}$ is regular because $f_{i}$ is a unit there. On the other hand, away from $\operatorname{supp}(X)$, $f_{i}+f_{i}^{\prime} \delta$ is a section of $\mathscr{A}_{X} \Leftrightarrow f_{i}^{\prime}$ is regular. This completes the proof of the claim.

Immediately we get

Corollary. Assume that $H^{1}\left(C, \mathcal{O}_{O}\left(D_{0}+D_{\infty}\right)\right)$ is not zero and $\operatorname{char}(k) \nmid \operatorname{deg}(f)$. Then, for all but a finite number of points $c$ of $C$, the required extension $\tilde{f}$ does not exist if $X$ is the Schiffer variation $(1 / t)(d / d t)$ at the point $c$.

Proof. The rational differential $d f$ is not zero. In characteristic zero, this is true because $f$ is not constant. In characteristic $p$, it follows because $p$ does not divide the degree of $f$.

Thus for all but a finite number of points $c$ of $C, X(f)=(1 / t)(d f / d t)$ is a principal part at $c$ of order one. As $H^{1}\left(C, \mathscr{O}_{C}\left(D_{0}+D_{\infty}\right)\right) \neq 0$, by the previous remark, we know that almost of these principal parts are not zero. Hence our corollary follows from the proposition.

Now we will be done as soon as we finish the

Proof of the theorem. Let $g: \widetilde{C} \rightarrow M$ be a family of smooth complete curves where $M$ is an irreducible variety. We will also assume that, for each point $m$ of $M, g$ gives a universal deformation of the fiber $g^{-1}(m)$.

For some positive integer $e$, let $\operatorname{Div} \rightarrow M$ be the variety of effective relative divisors on $\widetilde{C} / M$ of degree $e$. Let $X$ be the subset $\left\{\left(D_{0}, D_{\infty}\right) \mid d D_{0} \sim d D_{\infty}\right\}$ of Div $X_{M}$ Div.

By the see-saw principle, $X$ is a closed subset. Consider the conditions $D_{0} \neq D_{\infty}$ and $H^{1}\left(C, O_{C}\left(D_{0}+D_{\infty}\right)\right) \neq 0$. The first condition defines an open subset of $X$ and the second condition defines a closed subset of $X$ by upper-semicontinuity. Let $Y$ be the subvariety of $X$ defined by these two conditions. We have a tautological morphism $\sigma: Y \rightarrow M$. The precise statement of the theorem is that $\sigma$ omits an open dense subset of $M$.

Assume otherwise. Then by Sard's theorem (char $k=0$ ) we may find a locally closed smooth subvariety $M^{\prime}$ of $Y$, which is etale over 
$M$. Replacing $M$ by $M^{\prime}$ and $\widetilde{C}$ by $\widetilde{C} \times_{M} M^{\prime}$, we may assume that we have effective relative divisors $\widetilde{D}_{0}$ and $\widetilde{D}_{\infty}$ on $C$, whose fibers over $M$ satisfy the above two conditions. We may find effective relative divisors $\bar{E}, \bar{D}_{0}$ and $\bar{D}_{\infty}$ on $\widetilde{C}$ such that $\bar{D}_{0}$ and $\bar{D}_{\infty}$ have no common component and $\bar{E}+\bar{D}_{i}=\widetilde{D}_{i}$. Further replacing $M$ by an open subset we may assume that $\bar{D}_{0}$ and $\bar{D}_{\infty}$ are disjoint.

By construction we have a rational function $\tilde{f}$ on $\widetilde{C}$ with zero divisor $d \bar{D}_{0}$ and polar divisor $d \bar{D}_{\infty}$. By the first condition $\bar{D}_{0}$ and $\bar{D}_{\infty}$ are relative divisors of positive degree and consequently $\tilde{f}$ is not constant. We may now specialize our attention to a particular fiber $C$ of $g$. By the corollary and the universality of the deformation, $H^{1}\left(C,\left.\mathscr{O}_{\widetilde{c}}\left(\bar{D}_{0}+\bar{D}_{\infty}\right)\right|_{c}\right)=0$. Hence its quotient $H^{1}\left(C,\left.\mathscr{O}_{\widetilde{\sigma}}\left(\widetilde{D}_{0}+\widetilde{D}_{\infty}\right)\right|_{c}\right)=0$. As this violates the second condition, the theorem follows by contradiction.

\section{REFERENCES}

1. E. Arbarello and M. Cornalba, Su una congettura di Petri, to appear.

2. H. M. Farkas, Special divisors and analytic subloci of the Teichmüller space, Amer.

J. Math., 88 (1966), 881-901.

3. G. Kempf, On algebraic curves, J. für reìne und and. Math., 295 (1977), 40-48.

Received January 30, 1980. Research supported by NSF Grant \#7900965.

THE Johns Hopkins UNIVERSITY

BALTIMORE, MD 21218 



\section{PACIFIC JOURNAL OF MATHEMATICS}

EDITORS

DONALD BABBITT (Managing Editor)

University of California

Los Angeles, CA 90024

Hugo RossI

University of Utah

Salt Lake City, UT 84112

C. C. MOORE and ANDREW OGG

University of California

Berkeley, CA 94720

\section{J. DugundjI}

Department of Mathematics

University of Southern California

Los Angeles, CA 90007

R. FINN and J. MILGRAM

Stanford University

Stanford, CA 94305

\section{ASSOCIATE EDITORS}
R. ARENS
E. F. BECKENBACH
B. H. NEUManN
F. WOLF
K. YoSHIDA

\section{SUPPORTING INSTITUTIONS}

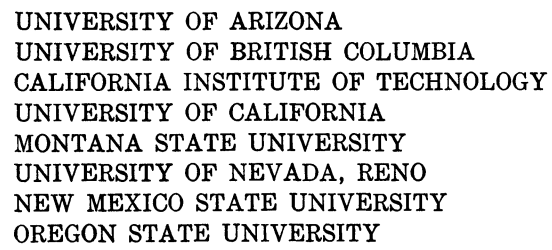

UNIVERSITY OF ARIZONA

UNIVERSITY OF BRITISH COLUMBIA

CALIFORNIA INSTITUTE OF TECHNOLOGY

UNIVERSITY OF CALIFORNIA

MONTANA STATE UNIVERSITY

UNIVERSITY OF NEVADA, RENO

NEW MEXICO STATE UNIVERSITY

OREGON STATE UNIVERSITY

\author{
UNIVERSITY OF OREGON \\ UNIVERSITY OF SOUTHERN CALIFORNIA \\ STANFORD UNIVERSITY \\ UNIVERSITY OF HAWAII \\ UNIVERSITY OF TOKYO \\ UNIVERSITY OF UTAH \\ WASHINGTON STATE UNIVERSITY \\ UNIVERSITY OF WASHINGTON
}

The Supporting Institutions listed above contribute to the cost of publication of this Journal, but they are not owners or publishers and have no responsibility for its content or policies.

Mathematical papers intended for publication in the Pacific Journal of Mathematics should be in typed form or offset-reproduced, (not dittoed), double spaced with large margins. Please do not use built up fractions in the text of the manuscript. However, you may use them in the displayed equations. Underline Greek letters in red, German in green, and script in blue. The first paragraph or two must be capable of being used separately as a synopsis of the entire paper. Please propose a heading for the odd numbered pages of less than 35 characters. Manuscripts, in triplicate, may be sent to any one of the editors. Please classify according to the scheme of Math. Reviews, Index to Vol. 39. Supply name and address of author to whom proofs should be sent. All other communications should be addressed to the managing editor, or Elaine Barth, University of California, Los Angeles, California, 90024.

50 reprints to each author are provided free for each article, only if page charges have been substantially paid. Additional copies may be obtained at cost in multiples of 50 .

The Pacific Journal of Mathematics is issued monthly as of January 1966. Regular subscription rate: $\$ 102.00$ a year (6 Vols., 12 issues). Special rate: $\$ 51.00$ a year to individual members of supporting institutions.

Subscriptions, orders for numbers issued in the last three calendar years, and changes of address shoud be sent to Pacific Journal of Mathematics, P.O. Box 969, Carmel Valley, CA 93924, U.S.A. Old back numbers obtainable from Kraus Periodicals Co., Route 100, Millwood, NY 10546.

\section{PUBLISHED BY PACIFIC JOURNAL OF MATHEMATICS, A NON-PROFIT CORPORATION}

Printed at Kokusai Bunken Insatsusha (International Academic Printing Co., Ltd.). 8-8, 3-chome, Takadanobaba, Shinjuku-ku, Tokyo 160, Japan. 


\section{Pacific Journal of Mathematics}

\section{Vol. 97, No. $2 \quad$ February, 1981}

Patrick Robert Ahern and N. V. Rao, A note on real orthogonal measures . . . . . 249

Kouhei Asano and Katsuyuki Yoshikawa, On polynomial invariants of fibered

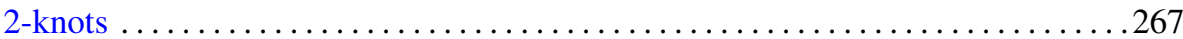

Charles A. Asmuth and Joe Repka, Tensor products for $S L_{2}(\mathscr{K})$. I.

Complementary series and the special representation

Gary Francis Birkenmeier, Baer rings and quasicontinuous rings have a

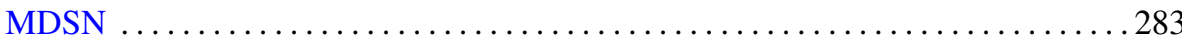

Hans-Heinrich Brungs and Günter Törner, Right chain rings and the generalized

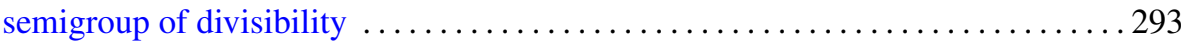

Jia-Arng Chao and Svante Janson, A note on $H^{1} q$-martingales . . . . . . . . . 307

Joseph Eugene Collison, An analogue of Kolmogorov's inequality for a class of

additive arithmetic functions

Frank Rimi DeMeyer, An action of the automorphism group of a commutative ring on its Brauer group

H. P. Dikshit and Anil Kumar, Determination of bounds similar to the Lebesgue

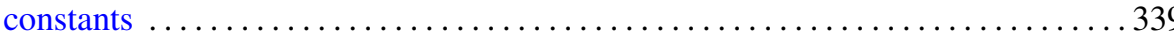

Eric Karel van Douwen, The number of subcontinua of the remainder of the plane

D. W. Dubois, Second note on Artin's solution of Hilbert's 17th problem. Order spaces

Daniel Evans Flath, A comparison of the automorphic representations of GL(3) and its twisted forms

Frederick Michael Goodman, Translation invariant closed $*$ derivations

Richard Grassl, Polynomials in denumerable indeterminates

K. F. Lai, Orders of finite algebraic groups

George Kempf, Torsion divisors on algebraic curves

Arun Kumar and D. P. Sahu, Absolute convergence fields of some triangular matrix methods

Elias Saab, On measurable projections in Banach spaces

Chao-Liang Shen, Automorphisms of dimension groups and the construction of AF algebras

Barry Simon, Pointwise domination of matrices and comparison of $\Phi_{p}$ norms

Chi-Lin Yen, A minimax inequality and its applications to variational inequalities

Stephen D. Cohen, Corrections to: "The Galois group of a polynomial with two indeterminate coefficients"

Phillip Schultz, Correction to: "The typeset and cotypeset of a rank 2 abelian group"

Pavel G. Todorov, Correction to: "New explicit formulas for the $n$th derivative of composite functions"

Douglas S. Bridges, Correction to: "On the isolation of zeroes of an analytic function" 